\title{
Preparation and Properties of Polyaniline in the Presence of Trehalose
}

\author{
Hirotsugu Kawashima, Hiromasa Goto* \\ Institute of Materials Science, Graduate School of Pure and Applied Sciences, University of Tsukuba, Tsukuba, Japan. \\ Email: gotoh@ims.tsukuba.ac.jp
}

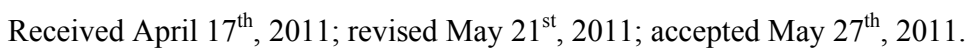

\begin{abstract}
Oxidative polymerization of aniline in aqueous solution in the presence of trehalose was conducted. Fourier transform infrared spectroscopy confirmed successful preparation of polyaniline containing a trace amount of trehalose. Electron spin resonance spectroscopy revealed that electron spin concentration of the polyaniline increases with aniline/trehalose ratio in the polymerization. Scanning electron microscopy revealed that the polyaniline shows granular and porous morphology. Electrical conductivity of these polyanilines was in the order of $10^{-4} \mathrm{~S} / \mathrm{cm}$.
\end{abstract}

Keywords: Conductive Polymer, Polyaniline, Trehalose

\section{Introduction}

A large variety of conjugated organic compounds has been prepared and studied because of their unique optical, electrical, and electronic properties [1-3]. Polyaniline (PANI) is one of the most promising conducting polymers because of its environmental stability, protonationadjustable electro-optical properties, and high conductivity [4-6]. Furthermore, preparation of PANI offers an advantage in environmental affinity because PANI can be synthesized in aqueous solution [7]. Recently, conductive polymer composites have attracted much attention because of their variety of properties and functionalities, such as mechanical strength, processibility [8-10]. In this research, we focused on employment of trehalose for the production of PANI.

Chemical structure of trehalose is illustrated in Figure 1. A number of organisms such as plants, insects, and microbes have trehalose [11]. It has been considered that trehalose plays important roles for life activity, especially in hibernation. Recently, a mass production technique of trehalose has been developed by the Hayashibara Co. (Okayama, Japan) by a biotechnology process [12]. To date, studies on trehalose are being conducted in several areas, such as food science, medical sciences, and pharmaceutical sciences $[13,14]$.

Here we report oxidative polymerization of aniline in the presence of trehalose. Both trehalose and aniline are soluble in water. This property allows a facile in-situ po- lymerization of aniline in the presence of trehalose by the typical method for preparation of PANI. In this research, ultraviolet-visible (UV-vis) absorption, Fourier transform infrared (FTIR) absorption spectroscopy, electron spin resonance (ESR) spectroscopy, scanning electron microscopy (SEM) observation, and electrical conductivity measurements of the PANIs were examined for the PANIs thus synthesized.

\section{Experimental Section}

\subsection{Materials}

Trehalose was purchased from Hayashibara (Okayama, Japan). Aniline (Tokyo Kasei) and water were distilled prior to use. Ammonium persulfate (APS, Kanto Chemicals) was used as an oxidizing agent. Sulfuric acid (Kanto Chemicals) was employed for preparation of anilinesulfuric salt prior to the polymerization. $\mathrm{N}$-methyl-2pyrrolidone (NMP, Kanto Chemicals) was used for UVvis measurement of the PANIs.

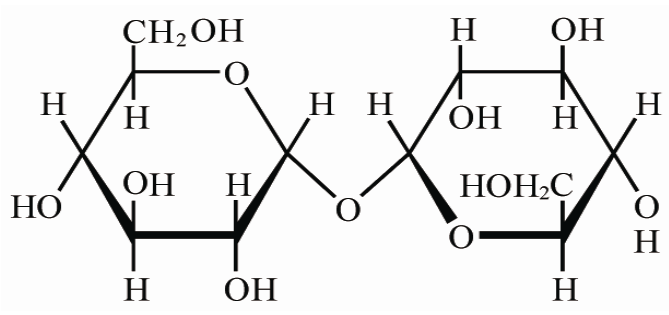

Figure 1. Chemical structure of trehalose. 
Table 1. Preparation of PANI/trehalose and PANI/(+)-CSA.

\begin{tabular}{|c|c|c|c|c|c|}
\hline Entry & aniline $(\mathrm{g})$ & trehalose $(\mathrm{g})$ & $\begin{array}{l}\text { trehalose/ } \\
\text { aniline }\end{array}$ & $\begin{array}{l}\text { recovered } \\
\text { amount }(\mathrm{g})\end{array}$ & color \\
\hline PANI1 & 0.05 & 5 & 100 & 0.02 & Russet \\
\hline PANI2 & 0.25 & 5 & 20 & 0.06 & Russet \\
\hline PANI3 & 0.5 & 5 & 10 & - & Russet \\
\hline PANI4 & 1.25 & 5 & 4 & 1.09 & $\begin{array}{l}\text { Dark } \\
\text { green }\end{array}$ \\
\hline PANI5 & 2.5 & 5 & 2 & 1.87 & $\begin{array}{l}\text { Dark } \\
\text { green }\end{array}$ \\
\hline PANI6 & 5 & 5 & 1 & 1.94 & $\begin{array}{l}\text { Dark } \\
\text { green }\end{array}$ \\
\hline PANI $^{\mathrm{a}}$ & 5 & 5 & 1 & 0.45 & Brown \\
\hline $\mathrm{PC}^{\mathrm{b}}$ & 1 & 0 & - & - & $\begin{array}{l}\text { Dark } \\
\text { green }\end{array}$ \\
\hline
\end{tabular}

${ }^{a}$ sulfuric acid was not used in the polymerization; ${ }^{b}(+)-10$-Camphorsulfonic acid ((+)-CSA) is employed instead of sulfuric acid.

\subsection{Equipment}

FTIR absorption spectra for the PANIs were measured with an FT/IR-300 spectrometer (Jasco) with the $\mathrm{KBr}$ method. UV-vis absorption spectra were recorded on a V-630 UV-vis optical absorption spectrometer (Jasco). ESR spectra were taken at room temperature using a JES-TE200 ESR spectrometer (JEOL). Surface images of PANIs were obtained with SEM (JSM-5610, JEOL). Electrical conductivity of the polymers was obtained by the dc four-probe method (Mitsubishi Lowrester-GP MCTP-T 610 with MCP-TP06P probe) at room temperature. A thermographic image of the PANI pallet was obtained with FLIR i5 (FLIR Systems).

\subsection{Synthesis}

Trehalose (5.0 g) and aniline (0.05 - $5.0 \mathrm{~g}$, Table 1) were dissolved in distilled water $(170 \mathrm{~mL})$. Sulfuric acid $(5.0$ $\mathrm{mL}, 95.0 \%$ ) was added to the reaction mixture except for preparation of PANI7 (without use of sulfuric acid in the polymerization). The mixture was then cooled to $\sim 0^{\circ} \mathrm{C}$ in an ice bath followed by addition of APS $(5.0 \mathrm{~g})$ in water $(30 \mathrm{~mL})$ to initiate oxidative polymerization, as shown in Scheme 1. After $24 \mathrm{~h}$, the solution was filtered, washed with distilled water $(200 \mathrm{~mL})$ for $12 \mathrm{~h}$ to remove trehalose and excess APS. Then, the solution was filtered again, washed with methanol $(200 \mathrm{~mL})$ for $12 \mathrm{~h}$. After filtration, the PANI was dried in a vacuum to yield deep emerald russet (PANI1, PANI2, and PANI3), deep emerald green (PANI4, PANI5, and PANI6), and brown (PANI7) solids. Quantities of the resultant polymers are summarized in Table 1. We also conducted preparation of PANI with (+)-10-camphorsulfonic acid $((+)$-CSA, (abbreviated PC), and a normal PANI (abbreviated PN) prepared by typical methods as references $[15,16]$. PANI1, PANI2, and PANI3 were obtained in trace

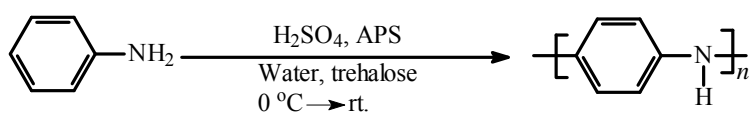

Scheme 1. Oxidative polymerization of aniline.

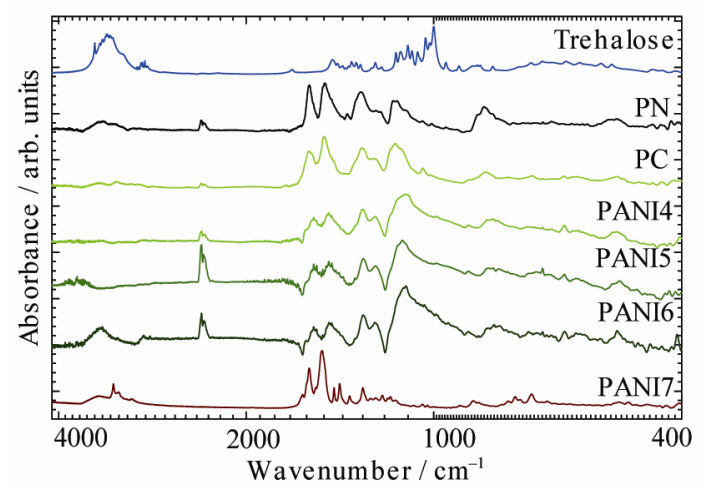

Figure 2. Fourier transform infrared (FTIR) spectra of trehalose, PN, PC, PANI4, PANI5, PANI6, and PANI7.

amounts. Therefore, we examined analysis of PANI4, PANI5, PANI6, and PANI7 samples.

\section{Results and Discussion}

Visual inspection of the samples color implies that these PANIs can be an emeraldine salt. FTIR measurements were examined for PANI4-PANI7, PC, and PN to confirm the chemical structure (Figure 2). Typical absorption bands of PANI are observed at $1558 \mathrm{~cm}^{-1}$ (quinoid (Q) $\mathrm{C}=\mathrm{C}$ stretching), $1472 \mathrm{~cm}^{-1}$ (benzenoid $\mathrm{C}=\mathrm{C}$ stretching), $1297 \mathrm{~cm}^{-1}\left(\mathrm{C}-\mathrm{N}\right.$ stretching), and $1119 \mathrm{~cm}^{-1}$ $(\mathrm{N}=\mathrm{Q}=\mathrm{N})$ [17]. Furthermore, PANI4, PANI5, PANI6, and trehalose have absorption band at $1100 \mathrm{~cm}^{-1}$. On the other hand, PANI7, PN, and PC have no absorption band in this region. This result implies that a small amount of trehalose was coated by PANI during polymerization for PANI4, PANI5, and PANI6. Here, hydrogen bonding interactions may exist between trehalose and PANI. As for PC, characteristic bands at $1150 \mathrm{~cm}^{-1}$ derived from a $\mathrm{C}-\mathrm{SO}^{3-}$ vibration of (+)-CSA appeared. This result confirms formation of the PANI/(+)-CSA.

PANI4, PANI5, and PANI6 have good solubility in NMP. The PANIs in NMP solution exhibit transparent blue color. CIE color space chromaticity confirms the color of the polymer in the solution, as shown in Figure 3. UV-vis optical absorption spectra of PANI4, PANI5, PANI6, and PANI7 in NMP are shown in Figure 4. An absorption band appears at $340 \mathrm{~nm}$ for PANI4, PANI5, and PANI6 due to $\pi-\pi^{*}$ transition of the main chain. The absorption band at $640 \mathrm{~nm}$ was assignable to charge carriers, so-called polarons (doping band).

These are typical absorption shapes for the doped state 
of PANI. As for PANI7, an absorption band at $380 \mathrm{~nm}$ appears. This is a typical absorption for aniline oligomers. This result indicates that PANI7 forms no well-developed main chain.

Generally, PANI prepared in acidic conditions is in the half-protonated state. In this case, PANI has charge carriers (polarons) along the backbone and exhibits electrical conductivity. ESR measurements revealed the existence of the radicals as the charge carriers in these polymers. ESR spectra of PANI4, PANI5, and PANI6 are shown in Figure 5. $g$-value, line-width of the signal, and spin concentration of the PANIs are summarized in Table 2.

The line shapes of these ESR signals are symmetric Lorentz patterns. Typical sharp signals for PANI4 are derived from well-developed delocalized polarons along the main chain. In the case of PANI5 and PANI6, line shapes are broadened and the $g$-value shifts slightly to

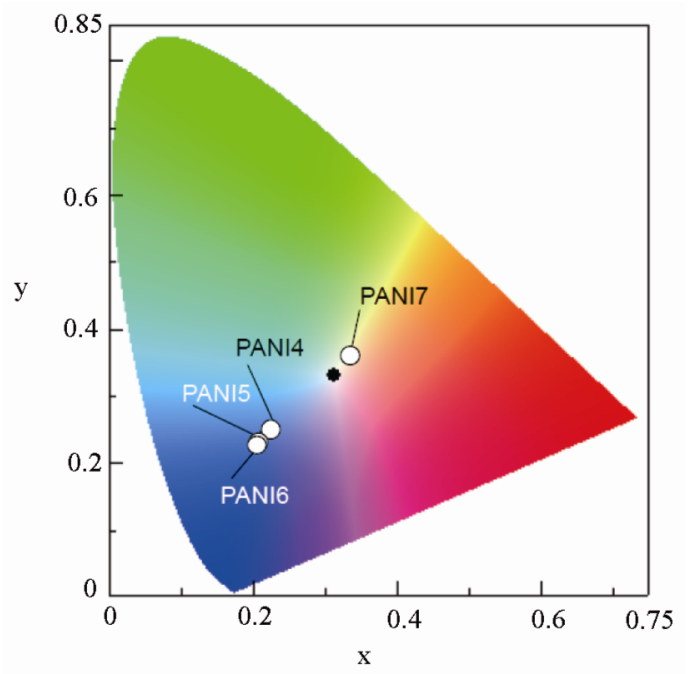

Figure 3. CIE color space chromaticity diagram of PANI4, PANI5, PANI6, and PANI7.

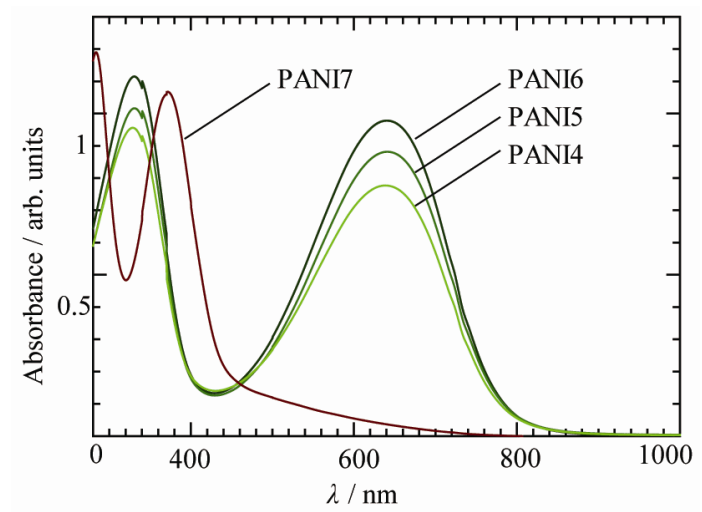

Figure 4.Ultraviolet-visible (UV-vis) absorption spectra of PANI4, PANI5, PANI6, and PANI7.

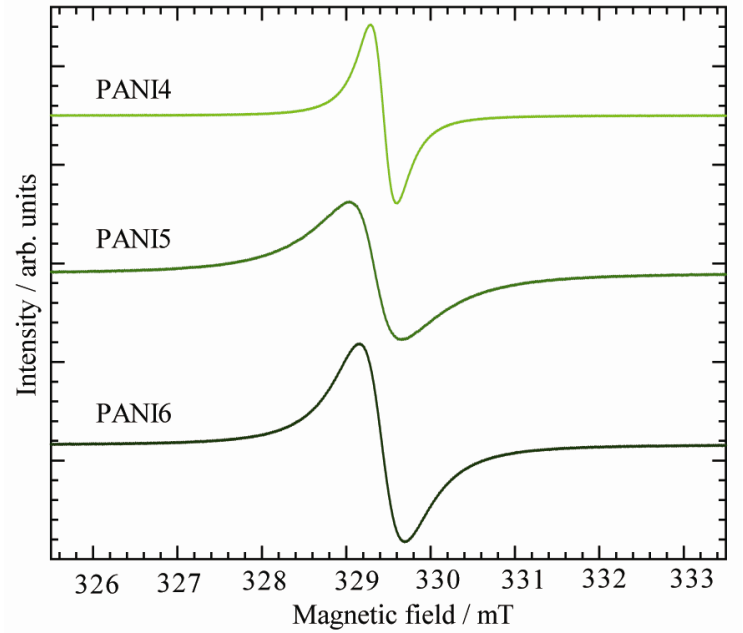

Figure 5. Electron spin resonance (ESR) spectra of PANI4, PANI5, and PANI6.

Table 2. ESR results and electrical conductivity.

\begin{tabular}{cccccc}
\hline Polymer & $\begin{array}{c}\text { Trehalose/ } \\
\text { Aniline }\end{array}$ & $g$-value & $\Delta H_{p p}(\mathrm{mT})$ & $N_{\mathrm{s}}$ (spins/g) & $\sigma(\mathrm{S} / \mathrm{cm})$ \\
\hline PANI4 & 4 & 2.0041 & 0.313 & $1.86 \times 10^{20}$ & $8.5 \times 10^{-4}$ \\
PANI5 & 2 & 2.00398 & 0.62 & $1.89 \times 10^{20}$ & $6.8 \times 10^{-4}$ \\
PANI6 & 1 & 2.004 & 0.527 & $2.22 \times 10^{20}$ & $5.3 \times 10^{-4}$ \\
\hline
\end{tabular}

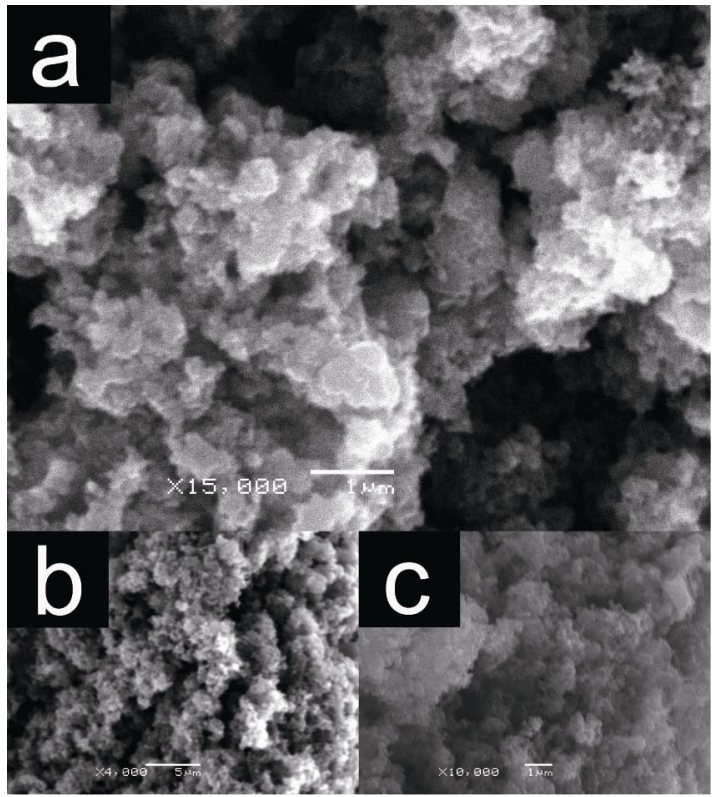

Figure 6. Scanning electron microscope (SEM) images of PANI4 (a), PANI5 (b), and PANI6 (c).

lower field. This suggests that bipolarons were also generated in PANI5 and PANI6. Spin concentration of these PANIs increase with aniline/trehalose ratio in the polymerization. 


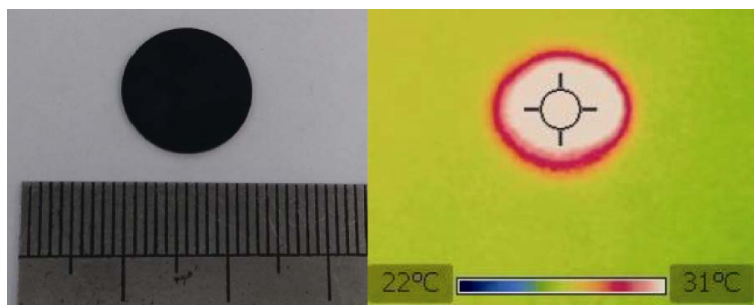

Figure 7. Compressed pellet of PANI6 (left) and thermography (right) under IR light irradiation.

Electrical conductivities of PANI4, PANI5, and PANI6 were examined using the four-probe method. These results are summarized in Table 2. Sample pellets of the PANIs for the measurement were prepared by pressing (100 MPa). Conductivity of these PANIs was increased with trehalose/aniline ratio in the polymerization. Conductivities of these PANIs thus prepared are comparable to typical PANI.

SEM images of PANI4, PANI5, and PANI6 show granular and macroporous structure (Figure 6). This porous morphology might provide gas adsorption functionality. Characteristic structure such as fiber structure, is often observed for PANIs [18]. However, the PANIs prepared in this study show no such fibril structure, but rather porous structure. This may be due to the fact that trehalose interrupts aggregation of PANI chains for formation of fibril structure.

Figure 7 shows compressed pellet of PANI6 and its thermography under irradiation of IR light. This result demonstrated that the polymer have good processability to form the pellet and infrared absorption nature derived from $\pi$-conjugation of the main chain.

\section{Conclusions}

A series of PANIs was prepared by oxidative polymerization of aniline in the presence of trehalose. The trehalose surface may play a role of a reaction field in the water medium during the polymerization. FTIR measurement indicated a trace amount of trehalose was coupled with PANI via hydrogen bonding. Polymerization of aniline in the presence of natural compounds allows tuning of electronic properties and morphology of the resultant PANI. This study might open the possibility of preparation of polyaniline in the presence of biological materials.

\section{REFERENCES}

[1] T. Kogej, F. Meyers, S. R. Marder, R. Silbey and J. L. Brédas, "Nonlinear Optical Properties of $\pi$-Conjugated Compounds Incorporating a Saturated Spacer Group," Synthetic Metals, Vol. 85, No. 1-3, 1997, pp. 1141-1142. doi:10.1016/S0379-6779(97)80185-3
[2] T. Higashihara, C.-L. Liu, W.-C. Chen and M. Ueda, "Synthesis of Novel $\pi$-Conjugated Rod-Rod-Rod Triblock Copolymers Containing Poly (3-hexylthiophene) and Polyacetylene Segments by Combination of Quasi-Living GRIM and Living Anionic Polymerization," Polymers, Vol. 3, No. 1, 2011, pp. 236-251. doi:10.3390/polym3010236

[3] K.-H. Yim, Z. Zheng, R. H. Friend, W. T. S. Huck and J.-S. Kim, "Surface-Directed Phase Separation of Conjugated Polymer Blends for Efficient Light-Emitting Diodes," Advanced Functional Materials, Vol. 18, Mo. 19, 2008, pp. 2897-2904.

[4] Y. Wang, H. D. Tran, L. Liao, X. Duan and R. B. Kaner, "Nanoscale Morphology, Dimensional Control, and Electrical Properties of Oligoanilines," Journal of American Chemical Society, Vol. 132, No. 30, 2010, pp. 10365 10373. doi:10.1021/ja1014184

[5] B. Bahmani, F. Moztarzadeh, M. Rabiee and M. Tahriri, "Development of an Electrochemical Sulfite Biosensor by Immobilization of Sulfite Oxidase on Conducting Polyaniline Film," Synthetic Metals, Vol. 160, No. 23-24, 2010, pp. 2653-2657.

doi:10.1016/j.synthmet.2010.10.020

[6] C. Chen and Y. Gao, "Electrosyntheses of Poly (Neutral Red), a Polyaniline Derivative," Electrochimicaacta, Vol. 52, No. 9, 2007, pp. 3143-3148. doi:10.1016/j.electacta.2006.09.056

[7] Y. Ma, S. R. Ali, L. Wang, P. L. Chiu, R. Mendelsohn and H. He, "In Situ Fabrication of a Water-Soluble, Self-Doped Polyaniline Nanocomposite: The Unique Role of DNA Functionalized Single-Walled Carbon Nanotubes," Journal of American Chemical Society, Vol. 128, No. 37, 2006, pp. 12064-12065. doi:10.1021/ja063375e

[8] X. Lu, Y. Yu, L. Chen, H. Mao, L. Wang, W. Zhang and Y. Wei, "Poly (Acrylic Acid)—Guided Synthesis of Helical Polyaniline Microwires," Polymer, Vol. 46, No. 14, 2005, pp. 5329-5333. doi:10.1016/j.polymer.2005.04.019

[9] Y. Yan, R. Wang, X. Qiu and Z. Wei, "Hexagonal Superlattice of Chiral Conducting Polymers Self-Assembled by Mimicking $\beta$-Sheet Proteins with Anisotropic Electrical Transport," Journal of American Chemical Society, Vol. 132, No. 34, 2010, pp. 12006-12012. doi:10.1016/j.polymer.2005.04.019

[10] R. Nagarajan, W. Liu, J. Kumar, S. K. Tripathy, F. F. Bruno and L. A. Samuelson, "Manipulating DNA Conformation Using Intertwined Conducting Polymer Chains," Macromolecules, Vol. 34, No. 12, 2001, pp. 3921-3927 doi:10.1016/j.polymer.2005.04.019

[11] M. Sakurai, T. Furuki, K. Akao, D. Tanaka, Y. Nakahara, T. Kikawada, M. Watanabe and M. T. Okuda, "Vitrification is Essential for Anhydrobiosis in An African Chironomid, Polypedilum Vanderplanki," Proceedings of the National Academy of Sciences of the United States of America, Vol. 105, No. 13, 2008, pp. 5093-5098. doi:10.1016/j.polymer.2005.04.019 
[12] T. Higashiyama, "Novel Functions and Applications of Trehalose," Pure and Applied Chemistry, Vol. 74, No. 7, 2002, pp. 1263-1269. doi:10.1351/pac200274071263

[13] D. Natan, A. Nagler and A. Arav, "Freeze-Drying of Mononuclear Cells Derived from Umbilical Cord Blood Followed by Colony Formation," PLoS ONE, Vol. 4, No. 4, 2009, p. e5240. doi:10.1371/journal.pone.0005240

[14] C. Rochelle and G. Lee, "Dextran or Hydroxyethyl Starch in Spray-Freeze-Dried Trehalose/Mannitol Microparticles Intended as Ballistic Particulate Carriers for Proteins," Journal of Pharmaceutical Sciences, Vol. 96, No. 9, 2007, pp. 2296-2309. doi:10.1002/jps.20861

[15] Y. Yan, Y. Zhang, W. Hu and Z. Wei, "Hierarchical
Crystalline Superstructures of Conducting Polymers with Homohelicity," Chemical European Journal, Vol. 16, No. 29, 2010, pp. 8626-8630. doi:10.1002/chem.201000999

[16] A. Andreatta, Y. Cao, J. C. Chiang, A. J. Heeger and P. Smith, "Electrically-Conductive Fibers of Polyaniline Spun from Solutions in Concentrated Sulfuric Acid," Synthetic Metals, Vol. 26, No. 4, 1988, pp. 383-389. doi:10.1016/0379-6779(88)90233-0

[17] N. Koji, "Infrared absorption spectra (Japanese)," Nankodo, Tokyo, 1960.

[18] J. Tang, X. Jing, B. Wang and F. Wang, "Infrared Spectra of Soluble Polyaniline," Synthetic Metals, Vol. 24, No. 3, 1988, pp. 231-238. doi:10.1016/0379-6779(88)90261-5 